\title{
ENHANCING SUSTAINABLE OIL PALM CULTIVATION USING COMPOST
}

\section{LAI GUAN YI*; SITI AISHAH ABD WAHID*; PREMKUMAR TAMILARASAN* and CHEAH SEE SIANG*}

\begin{abstract}
Increasing interest in the sustainability of oil palm cultivation has led to greater use of oil palm waste as a substitute for inorganic fertiliser and to improve soil health. Waste materials from oil palm mill process include palm oil mill effluent (POME), decanter cake and empty fruit bunches are co-composted. Co-composting these wastes would not only solve myriad of environmental issues, it could potentially improve soil quality and fresh fruit bunch production for sustainable palm oil production. Therefore, a field experiment was initiated to study the effects of compost and inorganic fertiliser on soil chemical properties and fresh fruit bunch yield of oil palm planted on a sedentary soil in Peninsula Malaysia. Application of either compost or fertiliser alone increased fresh fruit bunch yield, from 23.24 to 25.49 or 25.55 tFFB ha-1 $y^{-1}$. Soil chemical properties such as soil $\mathrm{pH}$, organic $C$, total $N$ and $P$, available $P$, exchangeable cations $\left(K^{+}\right.$, $\mathrm{Ca}^{2+}$ and $\mathrm{Mg}^{2+}$ ) and cation exchange capacity were significantly increased by the application of compost, making compost an effective organic material and nutrient source for improving the health of soils under oil palm cultivation.
\end{abstract}

Keywords: sustainable, compost, oil palm, nutrient, soil health, fresh fruit bunch, soil chemical properties.

Date received: 19 March 2019; Sent for revision: 29 April 2019; Received in final form: 19 June 2019 ; Accepted: 26 July 2019.

\section{INTRODUCTION}

Oil palm cultivation must be made more sustainable to ensure environmental protection is not compromised. Implementation of better, sustainable practices in the palm oil industry can lessen its impact on the ecosystem, without affecting profitability. The oil palm industry produces large amount of waste materials such as empty fruit bunches (EFB), palm oil mill effluent (POME) and decanter cake (Teh, 2016). These waste materials can be a major disposal nightmare if proper waste management was not carried out. Instead of disposing these materials, the industry can fully utilise them as an alternative,

\footnotetext{
* Plantation Research and Advisory,

Sime Darby Research Sdn Bhd,

Lot 2664, Jalan Pulau Carey,

42960 Carey Island, Selangor, Malaysia.

E-mail: lai.guan.yi@simedarbyplantation.com
}

renewable nutrient source for sustainable oil palm cultivation.

In Malaysia, there has been a great interest in converting oil palm waste materials into compost. Compost could potentially substitute inorganic fertilisers in oil palm cultivation, reducing the industry's dependence on an increasingly scarce and expensive nutrient source. Co-composting EFB, POME and decanter cake produces a chemically stable material that can be used as a source of nutrients (Silalahi et al., 2010) and for improving soil physico-chemical properties (Darmosarkoro and Sutarta, 2002). The nutrients available in the compost will be released gradually to the soil and eventually be taken up by oil palms. Thus, the use of compost as alternative nutrient source can reduce the cost of fertilisation and make palm oil production more profitable and sustainable (Tohiruddin and Foster, 2013). In view of the beneficial effects of using compost as alternative nutrient source, the 
Malaysian palm oil industry has started to explore this alternative approach to fertilisation (Teo et al., 2010).

This article reports the effect of compost compared to inorganic fertiliser on soil chemical properties and oil palm yield performance under Malaysian sedentary soils environment.

\section{MATERIALS AND METHOD}

Planting material used in this experiment was tenera palm (Dura x Pisifera) which was planted in 2006 with density of 144 palms ha-1 $^{-1}$ The experiment was, however, initiated in October 2013 when palms were 7 years old after planting (YAP).

The trial was laid out in Layang, Johor in Malaysia $\left(\mathrm{N}^{\circ} 1.810479 \mathrm{E}^{\circ} 103.457320\right)$ in a $4 \times 3$ factorial experiment arranged in randomised complete block design with three replications per treatment. Four levels of compost rates $\left(0,50,100\right.$ and $\left.150 \mathrm{~kg}_{\text {palm }}^{-1} \mathrm{yr}^{-1}\right)$ and three levels of fertiliser inputs $(0 \%, 50 \%$ and $100 \%$ of baseline fertiliser rate) were evaluated (Table 1). Ammonium chloride (AC, 25\% N) and Muriate of potash (MOP, $60 \% \mathrm{~K}_{2} \mathrm{O}$ ) were used as $\mathrm{N}$ and $\mathrm{K}$ sources, while Egyptian rock phosphate (ERP, 28\% $\mathrm{P}_{2} \mathrm{O}_{5}$ ) and kieserite (KIE, 27\% MgO) were used as $\mathrm{P}$ and $\mathrm{Mg}$ sources respectively. The baseline fertiliser rate used were $3.36 \mathrm{~kg} \mathrm{AC,} 3.75 \mathrm{~kg}$ MOP, $2.00 \mathrm{~kg}$ ERP and $1.25 \mathrm{~kg} \mathrm{KIE} \mathrm{palm}^{-1} \mathrm{yr}^{-1}$. Boron was applied at 0.1 $\mathrm{kg}$ palm ${ }^{-1} \mathrm{yr}^{-1}$ on a corrective basis.

Each plot consisted of $30(6 \times 5)$ palms with 12 (4x3) recording palms. The soil of the experimental plot was of the Harimau series (Typic Kandiudult). The physical properties of the soil at the study site contains an average 31\% clay, 3\% silt, 33\% fine sand and $29 \%$ coarse sand. The chemical composition of the soil before treatment application was $4.4 \mathrm{pH}$, $2.17 \%$ organic carbon, $0.14 \%$ total nitrogen, 354 ppm total phosphorus, 12 ppm available phosphorus, $0.14 \%$ exchangeable potassium, $0.84 \%$ exchangeable calcium, $0.25 \%$ exchangeable magnesium and 3.94 cation exchange capacity. Compost was applied as a single layer heap at the inter-palm area along planting row and it was applied once a year starting in 2013. AC and MOP were applied as two equal splits while rock phosphate and KIE were applied in single dosage, starting in 2013. All fertilisers were broadcast onto the compost heap except for nil compost plots where fertilisers were broadcast to the periphery of frond pile. Upkeep and maintenance of the experimental site was carried out in accordance to standard oil palm agricultural practices. The treatment details are shown in Table 1.

\section{Soil Analysis}

Annual soil analysis was carried out to assess soil nutrient status. Soil samples were collected from every recording palm in a plot, at the depth of $0-15 \mathrm{~cm}, 15-30 \mathrm{~cm}$ and $30-45 \mathrm{~cm}$ from the locations applied with compost. All soil samples collected in a plot were bulked as composite sample/depth/ location/plot. Soil samples were air-dried, ground and sieved ( $2 \mathrm{~mm}$ ) before subjecting to standard soil chemical analysis for $\mathrm{pH}$, organic $\mathrm{C}$ (Walkley and Black, 1934), total N (Kjeldahl method), total P (dilute double acid method using $\mathrm{H}_{2} \mathrm{SO}_{4}$ and $\mathrm{HCl}$ ), available P (Bray-Kurtz \#2), and cation exchange capacity $(\mathrm{CEC})$, exchangeable cations $(\mathrm{K}, \mathrm{Mg}$, and Ca) using leaching method.

\section{Compost Analysis}

The compost was made in-house from cocomposting EFB, POME and decanter cake for at least 45 days. Compost samples of approximately $50 \mathrm{~g}$ were collected from every compost heap in

TABLE 1. TREATMENT DETAILS

\begin{tabular}{|c|c|c|c|c|c|}
\hline \multirow{2}{*}{ Treatment } & \multirow{2}{*}{$\begin{array}{c}\text { Compost } \\
\left(k^{2} \text { palm }^{-1} \mathrm{yr}^{-1}\right)\end{array}$} & \multicolumn{4}{|c|}{ Fertiliser $\left(\mathrm{kg} \mathrm{palm}^{-1} \mathrm{yr}^{-1}\right)$} \\
\hline & & AC & MOP & ERP & Kieserite \\
\hline $\mathrm{T} 1$ & 0 & 0 & 0 & 0 & 0 \\
\hline $\mathrm{T} 2$ & 0 & 1.68 & 1.90 & 1.00 & 0.60 \\
\hline T3 & 0 & 3.36 & 3.75 & 2.00 & 1.25 \\
\hline $\mathrm{T} 4$ & 50 & 0 & 0 & 0 & 0 \\
\hline T5 & 50 & 1.68 & 1.90 & 1.00 & 0.60 \\
\hline T6 & 50 & 3.36 & 3.75 & 2.00 & 1.25 \\
\hline $\mathrm{T} 7$ & 100 & 0 & 0 & 0 & 0 \\
\hline T8 & 100 & 1.68 & 1.90 & 1.00 & 0.60 \\
\hline T9 & 100 & 3.36 & 3.75 & 2.00 & 1.25 \\
\hline T10 & 150 & 0 & 0 & 0 & 0 \\
\hline T11 & 150 & 1.68 & 1.90 & 1.00 & 0.60 \\
\hline T12 & 150 & 3.36 & 3.75 & 2.00 & 1.25 \\
\hline
\end{tabular}

Note: AC - ammonium chloride.

MOP - Muriate of potash.

ERP - Egyptian rock phosphate. 
each treatment plot and mixed thoroughly to form a composite sample $(600 \mathrm{~g})$ per treatment plot. All composite compost samples were weighed to obtain fresh weight followed by oven dry at $103 \pm 2^{\circ} \mathrm{C}$ for $3 \mathrm{hr}$ to obtain dry weight. All oven-dried samples were then subjected to standard chemical analysis for ash content, organic $\mathrm{C}, \mathrm{N}, \mathrm{P}, \mathrm{K}, \mathrm{Mg}$, and $\mathrm{B}$.

\section{Fresh Fruit Bunch (FFB) Yield Recording}

All fruit bunches were harvested at 10 - 12 days interval, starting in October 2013. Every bunch was weighed together with loose fruits using a Salter balance mounted on a tripod. All fruit bunches for each plot recorded in a year (October to September) were totalled. The total bunch weight was divided by the number of recording palms to obtain total bunch weight per palm which was then multiplied by the stand per hectare to obtain total bunch weight (FFB yield) per hectare per year.

FFB yield recorded in the first two years of treatment (October 2013 - September 2015) was omitted for statistical analysis. This is to minimise experimental error due to residual effect of previous fertiliser regime (Foster, 2003). FFB yield recorded in year three (October 2015 - September 2016) to year five (October 2017 - September 2018) were averaged and subjected to statistical analysis.

\section{Statistical Analysis}

All data were summarised according to treatments and subjected to statistical analysis using SAS JMP 9 (SAS Institute, 2010). The differences among treatment means were compared using Tukey's test and Least Significant Difference (LSD) test, at significance level of $5 \%$.

\section{EXPERIMENTAL RESULT}

\section{Nutrient Content of Compost and its Fertiliser Equivalent}

The moisture content, organic carbon, carbon:nitrogen $(\mathrm{CN})$ ratio and nutrient content of compost used in the experiment over 2014-2017 are depicted in Table 2. The compost used had an average moisture content of $57 \%$, organic $\mathrm{C}$ content of $41 \%$ and $\mathrm{CN}$ ratio of 33 . When the compost was applied at 50, 100 and $150 \mathrm{~kg}$ palm ${ }^{-1} \mathrm{yr}^{-1}$ respectively, it typically returned 0.22 to $0.65 \mathrm{~kg} \mathrm{~N}, 0.09$ to $0.29 \mathrm{~kg}$ $\mathrm{P}_{2} \mathrm{O}_{5^{\prime}} 0.75$ to $2.23 \mathrm{~kg} \mathrm{~K} \mathrm{O}$ and 0.10 to $0.30 \mathrm{~kg} \mathrm{MgO}$ palm ${ }^{-1} \mathrm{yr}^{-1}$ to the oil palm cultivation system. At the highest application rate of $150 \mathrm{~kg} \mathrm{palm}^{-1} \mathrm{yr}^{-1}$, the nutrients returned to the oil palm cultivation system was equivalent to $2.60 \mathrm{~kg} \mathrm{AC}, 1.04 \mathrm{~kg}$ ERP, $3.72 \mathrm{~kg}$ $\mathrm{MOP}$ and $1.11 \mathrm{~kg}$ kieserite palm ${ }^{-1} \mathrm{yr}^{-1}$, respectively.

\section{Soil Chemical Properties Three Years after Treatment}

Interaction effects. No significant interaction effects between compost and fertiliser on soil chemical properties were observed three years after treatment applications.

Soil $p H$. Compost and inorganic fertiliser both affected the soil $\mathrm{pH}$ significantly at all three soil depths (Table 3). Compost increased soil $\mathrm{pH}$ with increasing amount of compost applied to the soil while fertiliser decreased soil $\mathrm{pH}$ with increasing application rate. Increase in soil $\mathrm{pH}$ over a threeyear period can be seen with compost at all three depths, ranging from $13 \%$ to $28 \%$ with increasing application rate. Fertiliser application, however decreased soil $\mathrm{pH}$ over time (Table 3).

Soil organic carbon. Compost increased soil organic carbon with increasing amount of compost applied at all three soil depths, however, the effect was only significant at the highest application rate. In contrast, soil organic carbon decreased with increasing amount of fertiliser applied though the effect was not significant. Over a three-year period, application of compost increased soil organic carbon as high as $58 \%-68 \%$ at the highest application rate at all three depths. As opposed to compost, fertiliser application decreased soil organic carbon (Table 3).

Total nitrogen. Compost increased total nitrogen at all three soil depths with increasing amount of compost applied, however, the effect was only significant at the highest application rate. Fertiliser decreased total nitrogen with increasing application rate however the effect was not significant. Compost application increased total nitrogen over a three-

TABLE 2. MEAN (+/- SD) MOISTURE CONTENT, ORGANIC CARBON, CARBON:NITROGEN (CN) RATIO AND NUTRIENT CONTENTS OF COMPOST USED IN THE EXPERIMENT OVER 2014-2017

\begin{tabular}{|c|c|c|c|c|c|c|c|}
\hline \multirow{2}{*}{$\begin{array}{c}\text { Compost } \\
\left(\mathrm{kg} \mathrm{palm}^{-1} \mathrm{yr}^{-1}\right)\end{array}$} & \multirow{2}{*}{$\begin{array}{c}\text { Moisture } \\
\text { content }(\%)\end{array}$} & \multirow{2}{*}{$\begin{array}{c}\text { Organic C } \\
(\%)\end{array}$} & \multirow[t]{2}{*}{$\mathrm{CN}$ ratio } & \multicolumn{4}{|c|}{ Nutrient contents } \\
\hline & & & & $\mathbf{N}$ & $\mathbf{P}_{2} \mathbf{O}_{5}$ & $\mathrm{~K}_{2} \mathrm{O}$ & $\mathrm{MgO}$ \\
\hline 50 & $57 \pm 3.2$ & $41 \pm 2.5$ & $32 \pm 8.9$ & $0.22 \pm 0.05$ & $0.09 \pm 0.02$ & $0.75 \pm 0.15$ & $0.10 \pm 0.02$ \\
\hline 100 & $57 \pm 2.7$ & $40 \pm 2.2$ & $32 \pm 8.8$ & $0.42 \pm 0.12$ & $0.19 \pm 0.05$ & $1.45 \pm 0.36$ & $0.19 \pm 0.04$ \\
\hline 150 & $57 \pm 2.0$ & $41 \pm 2.0$ & $35 \pm 11.2$ & $0.65 \pm 0.20$ & $0.29 \pm 0.10$ & $2.23 \pm 0.34$ & $0.30 \pm 0.08$ \\
\hline Mean & $57 \pm 2.6$ & $41 \pm 2.2$ & $33 \pm 9.5$ & & & & \\
\hline
\end{tabular}


TABLE 3. MAIN EFFECTS OF COMPOST AND FERTILISER TREATMENTS ON SOIL $\mathrm{pH}$ AND ORGANIC CARBON AT THREE SOIL DEPTHS

\begin{tabular}{|c|c|c|c|c|c|c|c|}
\hline & \multirow{3}{*}{ Main effects } & \multicolumn{3}{|c|}{ Soil pH } & \multicolumn{3}{|c|}{ Organic carbon $(\%)$} \\
\hline & & \multicolumn{6}{|c|}{ 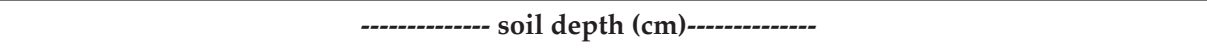 } \\
\hline & & 0-15 & $15-30$ & $30-45$ & $0-15$ & $15-30$ & $30-45$ \\
\hline \multirow{6}{*}{ 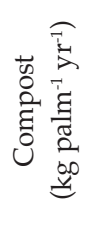 } & 0 & $4.3(-2)$ & $4.2(-2)$ & $4.1(-5)$ & $1.73(-28)$ & $1.63(-28)$ & $1.43(-40)$ \\
\hline & 50 & $5.1(13)$ & $5.1(13)$ & $4.9(10)$ & $2.58(18)$ & $2.61(14)$ & $2.26(16)$ \\
\hline & 100 & $5.2(21)$ & $5.2(21)$ & $5.1(19)$ & $2.97(39)$ & $3.17(48)$ & $2.79(35)$ \\
\hline & 150 & $5.6(28)$ & $5.4(27)$ & $5.3(21)$ & $3.36(58)$ & $3.41(61)$ & $3.22(68)$ \\
\hline & LSD $5 \%$ & 0.34 & 0.32 & 0.37 & 1.46 & 1.79 & 1.37 \\
\hline & Tukey 5\% & 0.46 & 0.43 & 0.50 & 1.95 & 2.40 & 1.8 \\
\hline \multirow{7}{*}{ 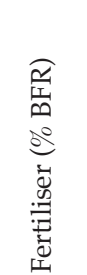 } & 0 & $5.5(26)$ & $5.3(23)$ & $5.2(20)$ & $3.30(48)$ & $3.35(44)$ & $3.05(49)$ \\
\hline & 50 & $5.0(14)$ & $5.0(14)$ & $4.9(13)$ & $2.41(9)$ & $2.56(21)$ & $2.34(18)$ \\
\hline & 100 & $4.7(6)$ & $4.7(8)$ & $4.5(2)$ & $2.27(3)$ & $2.20(2)$ & $1.89(-14)$ \\
\hline & LSD $5 \%$ & 0.30 & 0.28 & 0.32 & 1.26 & 1.55 & 1.19 \\
\hline & Tukey 5\% & 0.36 & 0.34 & 0.39 & 1.53 & 1.88 & 1.44 \\
\hline & CV $(\%)$ & 6.93 & 6.56 & 7.87 & 56.07 & 67.72 & 57.88 \\
\hline & SD & 0.74 & 0.74 & 0.74 & 1.70 & 2.08 & 1.76 \\
\hline
\end{tabular}

Note: () denotes percentage change between pre- and post-treatment.

$\mathrm{CV}$ - coefficient of variation.

LSD -Least Significant Difference.

SD - standard deviation.

year period to as high as $168 \%-205 \%$ at the highest application rate. Increase in total nitrogen was likewise seen in fertiliser treatments. However, the improvements in total nitrogen decreased with increasing fertiliser application rate (Table 4).

Total and available phosphorus. Compost and fertiliser increased total and available phosphorus at all three soil depths with increasing amount of compost and fertiliser applied. The effects of compost and fertiliser on available phosphorus was only significant at the highest application rate. The effect of fertiliser on soil total phosphorus was however not significant. Both compost and fertiliser increased total and available phosphorus over a three-year period (Table 5).

Exchangeable potassium and calcium. Compost increased exchangeable potassium and calcium significantly at all three soil depths with increasing amount of compost applied. However, fertiliser decreased exchangeable potassium and calcium with increasing application rate but the effect was not significant. Application of compost increased exchangeable potassium and calcium over a three year-period. Fertiliser application similarly showed improvements in exchangeable potassium and calcium, however, the improvements decreased with increasing rates (Table 6).

Exchangeable magnesium. Compost increased exchangeable magnesium significantly at all three soil depths but higher application rates of compost did not further increase exchangeable magnesium. Fertiliser increased exchangeable magnesium with increasing application rate but the effect was not significant. Both compost and fertiliser application increased exchangeable magnesium with increasing rates (Table 7).

CEC. Compost increased CEC at all three soil depths with increasing amount of compost applied. However, only the highest application rate increased CEC significantly. Contrary, fertiliser application did not affect CEC though CEC seemed to decrease with increasing application rate. CEC exchange capacity with compost application was subtle, however a 23\%-42\% increase in CEC can be seen at the highest application rate (Table 7).

\section{FFB Yield}

The main effect of treatment applications on FFB yield components are given in Table 8. The application of both compost and fertiliser increased FFB yield over a three-year period significantly. Application of both compost and fertiliser appeared to increase FFB yield through higher average bunch weight while bunch number was not affected (Table 8).

Application of $100 \mathrm{~kg}$ compost palm-1 $\mathrm{yr}^{-1}$ alone increased FFB yield from 23.24 to $25.55 \mathrm{t} \mathrm{FFB} \mathrm{ha}^{-1} \mathrm{yr}^{-1}$ over a three-year period, which was an increase by $2.31 \mathrm{t} \mathrm{FFB} \mathrm{ha}^{-1} \mathrm{yr}^{-1}$ over three years (Table 9). When fertiliser was applied alone, FFB yield responded significantly to applied fertiliser. Application of fertiliser at full baseline fertiliser rate increased FFB yield from 23.24 up to $25.49 \mathrm{t} \mathrm{FFB} \mathrm{ha}^{-1} \mathrm{yr}^{-1}$ over a three-year period, which was an increase by $2.25 \mathrm{t}$ FFB ha ${ }^{-1} \mathrm{yr}^{-1}$ over three years (Table 9). 
TABLE 4. MAIN EFFECTS OF COMPOST AND FERTILISER TREATMENTS ON SOIL TOTAL NITROGEN AT THREE SOIL DEPTHS

\begin{tabular}{|c|c|c|c|c|}
\hline & \multirow{3}{*}{ Main effects } & \multicolumn{3}{|c|}{ Total nitrogen $(\%)$} \\
\hline & & \multicolumn{3}{|c|}{------------ soil depth $(\mathrm{cm})$---------- } \\
\hline & & $0-15$ & $15-30$ & $30-45$ \\
\hline \multirow{6}{*}{ 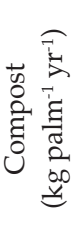 } & 0 & $0.196(28)$ & $0.168(23)$ & $0.155(15)$ \\
\hline & 50 & $0.307(103)$ & $0.305(112)$ & $0.248(88)$ \\
\hline & 100 & $0.343(142)$ & $0.348(143)$ & $0.317(152)$ \\
\hline & 150 & $0.408(168)$ & $0.399(188)$ & $0.371(205)$ \\
\hline & LSD 5\% & 0.15 & 0.14 & 0.13 \\
\hline & Tukey 5\% & 0.20 & 0.19 & 0.18 \\
\hline \multirow{7}{*}{ 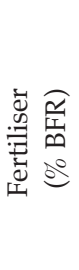 } & 0 & $0.374(149)$ & $0.354(158)$ & $0.336(156)$ \\
\hline & 50 & $0.298(91)$ & $0.303(110)$ & $0.257(99)$ \\
\hline & 100 & $0.270(89)$ & $0.258(85)$ & $0.226(80)$ \\
\hline & LSD $5 \%$ & 0.13 & 0.12 & 0.11 \\
\hline & Tukey 5\% & 0.15 & 0.15 & 0.14 \\
\hline & $\mathrm{CV}(\%)$ & 47.70 & 46.99 & 49.24 \\
\hline & SD & 0.18 & 0.19 & 0.18 \\
\hline
\end{tabular}

Note: () denotes percentage change between pre- and post-treatment.

$\mathrm{CV}$ - coefficient of variation.

LSD - Least Significant Difference.

SD - standard deviation.

TABLE 5. MAIN EFFECTS OF COMPOST AND FERTILISER TREATMENTS ON SOIL TOTAL AND AVAILABLE PHOSPHORUS AT THREE SOIL DEPTHS

\begin{tabular}{|c|c|c|c|c|c|c|c|}
\hline & \multirow{3}{*}{ Main effects } & \multicolumn{3}{|c|}{ Total P (ppm) } & \multicolumn{3}{|c|}{ Available P (ppm) } \\
\hline & & \multicolumn{6}{|c|}{ 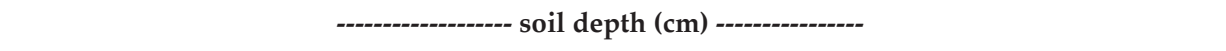 } \\
\hline & & 0-15 & $15-30$ & $30-45$ & 0-15 & $15-30$ & $30-45$ \\
\hline \multirow{6}{*}{ 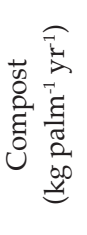 } & 0 & $454(34)$ & $424(36)$ & $361(14)$ & $36(242)$ & $33(282)$ & $34(334)$ \\
\hline & 50 & 714 (59) & $814(90)$ & 709 (103) & $126(446)$ & $132(621)$ & $132(942)$ \\
\hline & 100 & $661(81)$ & $754(100)$ & $627(93)$ & $110(1046)$ & $115(845)$ & 118 (1 236) \\
\hline & 150 & $776(104)$ & 766 (149) & 752 (153) & $158(1026)$ & 157 (1 336) & $166(1765)$ \\
\hline & $\mathrm{LSD} 5 \%$ & 198 & 244 & 202 & 73 & 75 & 79 \\
\hline & Tukey 5\% & 265 & 326 & 271 & 97 & 100 & 106 \\
\hline \multirow{7}{*}{ 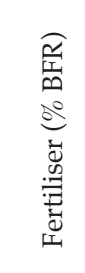 } & 0 & $612(43)$ & $597(55)$ & $531(60)$ & 47 (179) & $54(291)$ & $52(393)$ \\
\hline & 50 & 659 (67) & 703 (95) & $636(100)$ & $111(640)$ & 109 (657) & $111(1041)$ \\
\hline & 100 & 683 (109) & 769 (138) & $669(111)$ & 164 (1377) & 165 (1669) & $174(1971)$ \\
\hline & LSD 5\% & 171 & 211 & 175 & 63 & 65 & 69 \\
\hline & Tukey 5\% & 207 & 256 & 212 & 76 & 78 & 83 \\
\hline & $\mathrm{CV}(\%)$ & 31.02 & 36.17 & 33.80 & 69.11 & 70.02 & 72.06 \\
\hline & SD & 301 & 379 & 340 & 107 & 107 & 118 \\
\hline
\end{tabular}

Note: () denotes percentage change between pre- and post-treatment.

$\mathrm{CV}$ - coefficient of variation.

LSD - Least Significant Difference.

SD - standard deviation.

Almost similar FFB yield was obtained when compost and fertiliser were applied alone at 100 $\mathrm{kg}$ compost palm ${ }^{-1} \mathrm{yr}^{-1}$ and at full baseline fertiliser rate, respectively (Table 9). The amount of nutrients applied to the experimental palms in terms of fertiliser equivalent was however differed. At full baseline fertiliser rate, the applied AC, ERP, MOP and KIE were respectively 100\%, 194\%, 55\% and $79 \%$ higher than compost applied at $100 \mathrm{~kg} \mathrm{palm}^{-1}$ $\mathrm{yr}^{-1}$. The appreciable FFB yield response to lower nutrient input in the form of compost indicates that $\mathrm{N}, \mathrm{P}, \mathrm{K}$ and $\mathrm{Mg}$ in the compost were more effective than the nutrients in the fertiliser.

There was no significant interaction effect between compost and fertiliser on FFB yield (Table 9). However, the highest FFB yield of 26.21 t FFB $\mathrm{ha}^{-1} \mathrm{yr}^{-1}$ was obtained when full fertiliser rate was applied with $100 \mathrm{~kg}$ compost palm-1 $\mathrm{yr}^{-1}$. 
TABLE 6. MAIN EFFECTS OF COMPOST AND FERTILISER TREATMENTS ON SOIL EXCHANGEABLE POTASSIUM AND CALCIUM AT THREE SOIL DEPTHS

\begin{tabular}{|c|c|c|c|c|c|c|c|}
\hline & \multirow{3}{*}{$\begin{array}{c}\text { Main effects } \\
0-15\end{array}$} & \multicolumn{3}{|c|}{$\begin{array}{l}\text { Exchangeable K } \\
\text { (cmol kg-1 soil) }\end{array}$} & \multicolumn{3}{|c|}{$\begin{array}{l}\text { Exchangeable Ca } \\
\text { (cmol kg-1 soil) }\end{array}$} \\
\hline & & \multicolumn{6}{|c|}{--------------- soil depth $(\mathrm{cm})$-------------- } \\
\hline & & $15-30$ & $30-45$ & 0-15 & $15-30$ & $30-45$ & \\
\hline \multirow{6}{*}{ 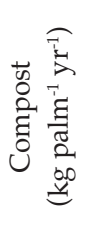 } & 0 & $0.19(43)$ & $0.19(46)$ & $0.19(30)$ & $0.55(-33)$ & $0.46(-37)$ & $0.33(-53)$ \\
\hline & 50 & $0.53(270)$ & $0.59(277)$ & $0.61(307)$ & $2.05(95)$ & $2.14(123)$ & $1.69(99)$ \\
\hline & 100 & $0.69(431)$ & $0.81(508)$ & $0.83(466)$ & $2.42(193)$ & $2.44(199)$ & $2.13(190)$ \\
\hline & 150 & $0.91(520)$ & $1.03(572)$ & $1.08(589)$ & $3.69(257)$ & $3.63(344)$ & $3.63(371)$ \\
\hline & LSD $5 \%$ & 0.25 & 0.27 & 0.30 & 0.11 & 1.27 & 1.37 \\
\hline & Tukey 5\% & 0.34 & 0.36 & 0.41 & 1.49 & 1.70 & 1.8 \\
\hline \multirow{7}{*}{ 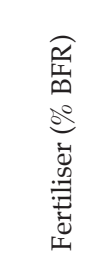 } & 0 & $0.71(366)$ & $0.80(416)$ & $0.81(406)$ & 2.64 (189) & $2.54(207)$ & $2.40(209)$ \\
\hline & 50 & $0.53(285)$ & $0.60(321)$ & $0.65(341)$ & $2.07(110)$ & $2.14(146)$ & 2.09 (187) \\
\hline & 100 & $0.50(300)$ & $0.57(330)$ & $0.57(300)$ & $1.83(104)$ & 1.83 (129) & $1.34(71)$ \\
\hline & LSD 5\% & 0.22 & 0.24 & 0.26 & 0.96 & 1.10 & 1.19 \\
\hline & Tukey 5\% & 0.26 & 0.29 & 0.32 & 1.17 & 1.33 & 1.44 \\
\hline & $\mathrm{CV}(\%)$ & 44.43 & 42.52 & 45.75 & 52.24 & 59.79 & 72.09 \\
\hline & SD & 0.42 & 0.47 & 0.52 & 1.68 & 1.78 & 1.95 \\
\hline
\end{tabular}

Note: () denotes percentage change between pre- and post-treatment.

$\mathrm{CV}$ - coefficient of variation.

LSD - Least Significant Difference.

SD - standard deviation.

TABLE 7. MAIN EFFECTS OF COMPOST AND FERTILISER TREATMENTS ON SOIL EXCHANGEABLE MAGNESIUM AND CATION EXCHANGE CAPACITY (CEC) AT THREE SOIL DEPTHS

\begin{tabular}{|c|c|c|c|c|c|c|c|}
\hline & \multirow[t]{3}{*}{ Main effects } & \multicolumn{3}{|c|}{$\begin{array}{l}\text { Exchangeable } \mathrm{Mg} \\
\text { (cmol kg-1 soil) }\end{array}$} & \multicolumn{3}{|c|}{$\begin{array}{c}\text { CEC } \\
\left(\mathrm{cmol} \mathrm{kg}^{-1} \text { soil) }\right.\end{array}$} \\
\hline & & \multicolumn{6}{|c|}{ 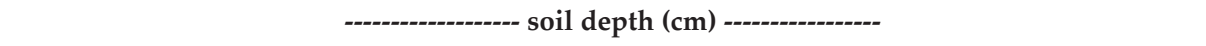 } \\
\hline & & 0-15 & $15-30$ & $30-45$ & 0-15 & $15-30$ & $30-45$ \\
\hline \multirow{6}{*}{ 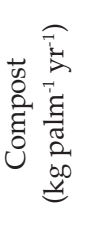 } & 0 & $0.11(-51)$ & $0.10(-47)$ & $0.09(-52)$ & $3.10(-29)$ & $3.21(-17)$ & $2.86(-26)$ \\
\hline & 50 & $1.86(500)$ & $1.67(476)$ & $1.26(425)$ & $4.52(8)$ & $4.66(22)$ & $3.71(-1)$ \\
\hline & 100 & 1.51 (439) & $1.54(492)$ & $1.23(443)$ & $4.46(17)$ & 3.95 (7) & $4.23(15)$ \\
\hline & 150 & $1.68(460)$ & $1.48(517)$ & $1.34(483)$ & $5.40(23)$ & $5.58(42)$ & $5.32(37)$ \\
\hline & LSD 5\% & 0.61 & 0.57 & 0.52 & 1.58 & 1.44 & 1.39 \\
\hline & Tukey 5\% & 0.82 & 0.76 & 0.69 & 2.11 & 1.93 & 1.86 \\
\hline \multirow{7}{*}{ 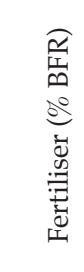 } & 0 & $1.06(296)$ & $0.87(263)$ & $0.77(246)$ & $4.75(11)$ & $4.63(16)$ & $4.51(18)$ \\
\hline & 50 & $1.26(310)$ & $1.24(372)$ & $1.12(421)$ & $4.08(-3)$ & $4.24(13)$ & $4.03(7)$ \\
\hline & 100 & $1.55(496)$ & $1.48(537)$ & $1.05(367)$ & $4.29(5)$ & $4.18(12)$ & $3.54(-7)$ \\
\hline & LSD 5\% & 0.53 & 0.49 & 0.45 & 1.37 & 1.25 & 1.20 \\
\hline & Tukey 5\% & 0.64 & 0.59 & 0.54 & 1.65 & 1.51 & 1.46 \\
\hline & $\mathrm{CV}(\%)$ & 48.42 & 48.21 & 54.24 & 36.89 & 33.85 & 35.29 \\
\hline & SD & 1.08 & 1.06 & 0.91 & 1.70 & 1.64 & 1.65 \\
\hline
\end{tabular}

Note: () denotes percentage change between pre- and post-treatment.

$\mathrm{CV}$ - coefficient of variation.

LSD - Least Significant Difference.

SD - standard deviation.

\section{Economic Analysis}

In this study, when fertiliser and compost were assumed to have equal efficiency in supplying nutrients to oil palm, compost was 2.4-fold more expensive than fertiliser (Table 10). Even when higher nutrient supply efficiency of compost was accounted for, the application cost of compost was still higher than fertiliser.

\section{DISCUSSION}

The compost derived from co-composting EFB, POME and decanter cake contains sufficient nutrients to meet palm demand of nutrients for optimum growth and production. The compost applied at $100 \mathrm{~kg}$ palm ${ }^{-1} \mathrm{yr}^{-1}$ could potentially substitute average inorganic fertiliser (baseline fertiliser rate used in the experiment) applied to 
TABLE 8. MAIN EFFECTS OF COMPOST AND FERTILISER TREATMENTS ON FFB YIELD COMPONENTS OVER A THREE-YEAR PERIOD

\begin{tabular}{|c|c|c|c|c|}
\hline & \multirow[b]{2}{*}{ Main effects } & \multicolumn{3}{|c|}{ Mean FFB yield of three harvesting years } \\
\hline & & $\begin{array}{c}\text { FFB } \\
\left(\mathrm{th} \mathbf{h}^{-1} \mathrm{yr}^{-1}\right)\end{array}$ & $\begin{array}{c}\text { NOB } \\
\left(\text { palm }^{-1} \mathrm{yr}^{-1}\right)\end{array}$ & $\begin{array}{c}\text { ABW } \\
\left(\mathrm{kg} \mathrm{palm}^{-1}\right)\end{array}$ \\
\hline \multirow{6}{*}{ 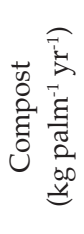 } & 0 & 24.09 & 8.47 & 19.85 \\
\hline & 50 & 24.00 & 8.35 & 19.97 \\
\hline & 100 & 25.55 & 8.76 & 20.33 \\
\hline & 150 & 25.72 & 8.86 & 20.18 \\
\hline & LSD 5\% & 1.20 & 0.54 & 0.79 \\
\hline & Tukey $5 \%$ & 1.61 & 0.73 & 1.06 \\
\hline \multirow{7}{*}{ 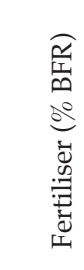 } & 0 & 24.28 & 8.87 & 19.04 \\
\hline & 50 & 24.32 & 8.20 & 20.61 \\
\hline & 100 & 25.92 & 8.75 & 20.60 \\
\hline & LSD 5\% & 1.04 & 0.47 & 0.69 \\
\hline & Tukey $5 \%$ & 1.26 & 0.57 & 0.83 \\
\hline & $\mathrm{CV}(\%)$ & 4.95 & 6.44 & 4.03 \\
\hline & SD & 1.85 & 0.70 & 1.14 \\
\hline
\end{tabular}

Note: NOB denotes number of fresh fruit bunches (FFB) and ABW denotes average bunch weight.

$\mathrm{CV}$ - coefficient of variation.

LSD - Least Significant Difference.

SD - standard deviation.

TABLE 9. THE COMPOST x FERTILISER EFFECT ON FFB YIELD OVER A THREE-YEAR PERIOD

\begin{tabular}{|c|c|c|c|}
\hline \multirow[t]{2}{*}{$\begin{array}{c}\text { Compost } \\
\left(\mathrm{kg} \mathrm{palm}^{-1} \mathrm{yr}^{-1}\right)\end{array}$} & \multicolumn{3}{|c|}{$\begin{array}{c}\text { Fertiliser } \\
(\% \text { of baseline fertiliser rate) }\end{array}$} \\
\hline & 0 & 50 & 100 \\
\hline & \multicolumn{3}{|c|}{ - } \\
\hline 0 & 23.24 & 23.53 & 25.49 \\
\hline 50 & 23.01 & 22.89 & 26.10 \\
\hline 100 & 25.55 & 24.89 & 26.21 \\
\hline 150 & 25.33 & 25.95 & 25.89 \\
\hline$p$-value & - & 0.3332 & - \\
\hline $\mathrm{CV}(\%)$ & - & 4.95 & - \\
\hline
\end{tabular}

Note: FFB - fresh fruit bunches.

$\mathrm{CV}$ - coefficient of variation.

TABLE 10. COST COMPARISON BETWEEN COMPOST AND INORGANIC FERTILISER

\begin{tabular}{|c|c|c|c|}
\hline \multirow[t]{2}{*}{ Fertilisers } & \multirow{2}{*}{$\frac{\text { At equal efficiency }}{\text { RM }}$} & \multicolumn{2}{|c|}{ At higher compost efficiency } \\
\hline & & Efficiency & RM \\
\hline Compost at $14.4 \mathrm{t} \mathrm{ha}^{-1} \mathrm{yr}^{-1}$ & 1152 & & 1152 \\
\hline Application costs per hectare & 422 & & 422 \\
\hline Total compost costs & 1574 & & 1574 \\
\hline Ammonium chloride $1.68 \mathrm{~kg} \mathrm{palm}^{-1} \mathrm{yr}^{-1}$ & 117 & $+100 \%$ & 235 \\
\hline Muriate of potash $2.42 \mathrm{~kg} \mathrm{palm}^{-1} \mathrm{yr}^{-1}$ & 429 & $+55 \%$ & 665 \\
\hline Rock phosphate $0.68 \mathrm{~kg} \mathrm{palm}^{-1} \mathrm{yr}^{-1}$ & 36 & $+194 \%$ & 107 \\
\hline Kieserite $0.70 \mathrm{~kg} \mathrm{palm}^{-1} \mathrm{yr}^{-1}$ & 59 & $+79 \%$ & 105 \\
\hline Application costs per hectare & 72 & - & 72 \\
\hline Total inorganic costs & 713 & & 1184 \\
\hline
\end{tabular}

Note: Fertiliser prices $\left(\mathrm{RM} \mathrm{t}^{-1}\right)$ : ammonium chloride $=485$, Muriate of potash $=1232$, Rock phosphate $=370$, Kieserite $=585$, compost $=80$.

Density per hectare $=144$ palms 
oil palm planted on sedentary soils in Peninsula Malaysia each year, except for $\mathrm{N}$ and $\mathrm{P}$ which are lower in compost. Tohiruddin and Foster (2013) reported that compost applied at $75 \mathrm{~kg}_{\text {palm }}{ }^{-1} \mathrm{yr}^{-1}$ could substitute average inorganic fertiliser (except for P) applied to oil palm planted on volcanic ash soil in Sumatera.

Inorganic fertiliser has been shown to increase FFB yield by supplying the required nutrients for palm growth and FFB production (Foster, 2003). This is further confirmed by the present study. However, the present study also revealed that inorganic fertiliser did little to improve overall soil health compared to compost. Malaysian sedentary soils are generally weathered, acidic in nature, with soil $\mathrm{pH}$ ranging from 4.0-4.9 (Hartley, 1988). Soil pH is widely accepted as an important factor regulating a range of soil biological and chemical processes. Application of inorganic fertiliser particularly $\mathrm{AC}$ decreased soil $\mathrm{pH}$ with increasing application rate, indicating that inorganic fertilisation caused soil acidification. Similar soil acidification effect of inorganic fertiliser on Malaysian soils were reported by Lim et al. (2002). Ammonium nitrogen $\left(\mathrm{NH}_{4}-\mathrm{N}\right)$ is known to decrease soil $\mathrm{pH}$ through leaching of basic cations and production of $\mathrm{H}^{+}$as a result of nitrification (Havlin et al., 1999). In this study, leaching of basic cations, increase in $\mathrm{H}^{+}$and the resultant exchange of cations with $\mathrm{NH}_{4}^{+}$were most likely responsible for the decrease in soil $\mathrm{pH}$ in fertilised plots. Compost, however, was able to raise soil $\mathrm{pH}$ by binding the positively-charged hydrogen ions, taking the $\mathrm{H}^{+}$ out of the solution (Pocknee and Sumner, 1997). The release of basic cations such as $\mathrm{K}^{+}, \mathrm{Ca}^{2+}, \mathrm{Mg}^{2+}$ after mineralisation of compost would have increased the base saturation of the soil solution that resulted in an increased soil $\mathrm{pH}$.

Soil organic carbon increased with the addition of compost. This is attributed to the return of organic matter to the soil after decomposition. Use of inorganic fertiliser, however, reduced soil organic carbon. The decrease in soil organic carbon may have been attributed to higher decomposition rate of soil organic matter as a result of higher input of readily available nitrogen released from ammonium chloride (Russell et al., 2005). It has been shown that fertiliser nitrogen enhances the activities of heterotrophic soil microorganisms that use carbon derived from crop residues and thus stimulate decomposition (Khan, 2007).

The addition of nitrogen through AC did not increase, but decreased soil total nitrogen with increasing application rate. Sandy Malaysian soils with low $\mathrm{pH}$ can cause appreciable loss of ammonium nitrogen through leaching (Foster, 1976). Compost, on the other hand, provided an excellent buffer to soil $\mathrm{pH}$, binding cations and hence reducing the rate of leaching. The soil of the study site contains an average $31 \%$ clay, 3\% silt,
$33 \%$ fine sand and 29\% coarse sand. The sandy and acidic conditions of the study site could have caused higher leaching losses of fertiliser nitrogen with increasing application rate.

In the present study, application of compost increased soil total and available P significantly. The increase in soil total $\mathrm{P}$ is likely attributed to addition of organic P contained in the compost. Increase availability of $\mathrm{P}$ in soil applied with compost is likely attributed to enhanced microbial activity during decomposition which indirectly leads to faster release of soluble $\mathrm{P}$ contained in the compost and rock phosphate, and a reduction in Al-P and Ca-P fractions (Ayaga et al., 2006; Budianta et al., 2010).

Similarly, soil applied with rock phosphate saw significant increase in soil available $\mathrm{P}$, however, soil total $P$ remained unchanged. Rock phosphate is not readily available for palm uptake and its dissolution rate is greatly influence by soil $\mathrm{pH}$ as reported by Chien et al. (2010). The low soil pH of the study site provides a favourable condition for the dissolution of rock phosphate applied, hence availability of $\mathrm{P}$ in soil applied with rock phosphate increases with increasing application rate.

The addition of compost significantly increased soil exchangeable cations and CEC. This can be explained by the increase in soil organic $C$ and the concomitant increase in soil $\mathrm{pH}$ and available cations due to compost addition. Enhancement of soil organic $C$ would increase soil humus or soil organic colloid which in turn increases soil CEC (Tan, 1994). Enhanced soil CEC would in turn increase soil nutrient holding capacity ( $\mathrm{Xu}$ et al., 2012). The higher CEC partly explained the increase in exchangeable cations in the compost-amended soil. Darmosarkoro and Sutarta (2002) also reported significant increases in soil exchangeable cations after compost application in Sumatra.

Application of compost and inorganic fertiliser increased FFB yield over a three-year period. Thus, further confirmed that additional nutrients either in the form of compost or inorganic fertiliser are needed to sustain optimum palm growth and FFB production for oil palm planted on highly weathered sedentary soils in Malaysia. However, the amount of nutrients needed to produce similar FFB yield at the study site was much lower for palms applied with compost compared to palms applied with inorganic fertiliser. The superior effect of compost on FFB yield performance was also observed in Sumatra by Tohiruddin and Foster (2013). They concluded that application of compost improved palm nutritional status due to improved soil chemical properties, which in turn gave higher FFB yield compared to inorganic fertiliser. They found that $\mathrm{N}, \mathrm{P}, \mathrm{K}$ and $\mathrm{Mg}$ in the compost were $66 \%, 37 \%, 20 \%$ and $20 \%$ more effective than the nutrients in the inorganic fertiliser. In this study, $\mathrm{N}$, $\mathrm{P}, \mathrm{K}$ and $\mathrm{Mg}$ in the compost were $100 \%, 194 \%, 55 \%$ 
and $79 \%$ more effective than the nutrients in the inorganic fertiliser.

Despite clear agronomic advantages, application of compost to oil palm was less profitable as shown in the present study. This is in contrast to Tohiruddin and Foster (2013) who found that applying $15 \mathrm{t}$ compost ha-1 $\mathrm{yr}^{-1}$ was more profitable than application of inorganic fertilisers at the same nutrient rates as $15 \mathrm{t}$ compost at $65 \%$ moisture content under Sumatran environments. One of the main factors contributed to the higher cost of compost application in the present study was the material cost of compost, which was two-fold more expensive than the compost used by Tohiruddin and Foster (2013).

\section{CONCLUSION}

Both compost and inorganic fertiliser increased FFB yields but application of inorganic fertiliser did little in improving soil physico-chemical properties. Apart from increasing FFB yield, application of compost improved soil chemical properties such as soil $\mathrm{pH}$, organic $\mathrm{C}$, total $\mathrm{N}$ and $\mathrm{P}$, available $\mathrm{P}$, exchangeable cations and CEC. On the other hand, application of inorganic fertiliser decreased soil $\mathrm{pH}$, organic $\mathrm{C}$, total $\mathrm{N}$ and exchangeable $\mathrm{K}$ and $\mathrm{Ca}$. The beneficial effects of compost on soil physicochemical properties were thus clearly demonstrated in the present study.

To produce the same level of FFB yield, a lower amount of nutrients in the form of compost is needed compared to nutrients in the form of inorganic fertiliser. Compost due to its impact on soil physico-chemical properties allows nutrients to be more efficiently supplied versus the manner in which nutrients is supplied by inorganic fertilisers. The greater nutrient supply efficiency of compost implies that the oil palm industry could lessen reliance on inorganic fertiliser and hence make oil palm cultivation more sustainable.

\section{ACKNOWLEDGMENT}

The authors would like to thank Sime Darby Plantation Berhad for permission to publish this article. Our appreciation also goes to the staff of Oil Palm Research Section for assistance rendered in carrying out research activities and collecting research data. Special thanks go to the staff and management of Layang Estate for their invaluable assistance and cooperation.

\section{REFERENCES}

Ayaga, G; Todd, A and Brookes, P C (2006). Enhanced biological cycling of phosphorus increase its availability to crops in low-input Sub-Saharan farming systems. Soil Biol. Biochem., 38: 81-90.

Budianta, D; A Halim, P K S; Midranisiah and Bolan, N S (2010). Palm oil compost reduces aluminium toxicity thereby increases phosphate fertiliser use efficiency in Ultisols. 19 $9^{\text {th }}$ World Congress of Soil Science, Soil Solutions for a Changing World. Brisbane, Australia. p. 221-223.

Chien, S H; Prochnow, L and Mikkelsen, R (2010). Agronomic use of phosphate rock for direct application. Better Crops Vol. 94 No. 4: 21-23.

Darmosarkoro, W and Sutarta, E S (2002). Application of EFB compost on acidic soil in North Sumatra to increase soil bases and decrease aluminium. Proc. of the International Oil Palm Conference 2002 (IOPC) - Agriculture Conference. Indonesian Oil Palm Research Institute (IOPRI), Nusa Dua Bali, Indonesia. p. 464-470.

Foster, H L (1976). Comparison of different fertiliser nutrient sources for oil palm: A review. MARDI Report No. 42: 1-8.

Foster, H L (2003). Assessment of oil palm fertiliser requirements. Oil Palm - Management for Large and Sustainable Yields (Fairhurst, T H and Härdter, $\mathrm{R}$ eds.). Potash \& Phosphate Institute of Canada (ESEAP), Singapore. p. 231-257.

Hartley, C W S (1988). The Oil Palm. $3^{\text {rd }}$ Edition. Longman, London/New York. 606 pp.

Havlin, J L; Beaton, J D; Tisdale, S L and Nelson, W L (1999). Soil Fertility and Fertilisers, An Introduction to Nutrient Management. Prentice Hall: Upper Saddle River, NJ, USA. p. 499.

Khan, S A; Mulvaney, R L; Ellsworth, T R and Boast, C W (2007). The myth of nitrogen fertilization for soil carbon sequestration. J. Environ. Qual., 36: 18211832.

Lim, K C and Zaharah, A R (2002). The effects of oil palm empty fruit bunches on oil palm nutritionand yield, and soil chemical properties. J. Oil Palm Res. Vol. 14 No. 2: 1-9.

Pocknee, S and Sumner, M E (1997). Cation and nitrogen contents of organic matter determine its soil liming potential. Soil Sci. Soc. Am. J., 61: 86-92.

Russell, A E; Laird, D A; Parkin, T B and Mallarino, A P (2005). Impact of nitrogen fertilization and cropping system on carbon sequestration in Midwestern Mollisols. Soil Sci. Soc. Am. J., 69: 413422. 
SAS Institute Inc. (2010). Using JMP 9. Cary, NC: SAS Institute Inc.

Silalahi, A J; Burden, J L and Foster, H L (2010). Economic benefits of using aerated bunkers to produce consistent high quality compost from palm oil mill by-products. Proc. of the International Oil Palm Conference (IOPC) 2010 - Chemistry and Technology Conference. Indonesian Oil Palm Research Institute (IOPRI). Yogyakarta, Indonesia. 12 pp.

Tan, K H (1994). Environmental Soil Science. Marcel Dekker, Inc. New York. 304 pp.

Teh, C B S (2016). Availability, use and removal of oil palm biomass in Indonesia. Working paper prepared for International Council on Clean Transportation (ICCT). Washington D.C., DC: International Council on Clean Transportation. $39 \mathrm{pp}$.
Teo, L; Muhammad Shukri, R; Ong, K P and Zainuriah, A (2010). Alternative oil palm fertilizer sources and management. Oil Palm Bulletin No. 61: 11-32.

Tohiruddin, L and Foster, H L (2013). Superior effect of compost derived from oil palm mill by-products as a replacement for inorganic fertilisers applied to oil palm. J. Oil Palm Res. Vol. 25: 123-137.

Walkley, A and Black, I A (1934). An examination of the Degtjareff method for determining soil organic matter and a proposed modification of the chromic acid titration method. Soil Sci., 37: 29-38.

Xu, G; Lv, Y; Sun, J; Shao, H and Wei, L (2012). Recent advances in biochar applications in agricultural soils: Benefits and environmental implications. CLEAN-Soil Air Water, 40: 1093-1098. 